\title{
Association between GSTP1 Genotypes and Hormone Receptor Phenotype in Invasive Ductal Carcinomas of Breast
}

\author{
Mohamad Nidal Khabaz ${ }^{1 *}$, Mamdooh Abdullah Gari², Jaudah Ahmed Al- \\ Maghrabi $^{3}$, Taoufik Nedjadi ${ }^{4}$, Marwan Bakarman ${ }^{5}$
}

\begin{abstract}
Eighty six cases of invasive ductal breast carcinomas were utilized to investigate GSTP1 polymorphisms in certain immunohistochemistry (IHC) subtypes of breast cancer with respect to ER, PR and HER2 expression. The frequency of wild allele homozygote, heterozygote and variant allele homozygote genotypes were $46.5 \%$, $52.3 \%$ and $1.16 \%$ respectively; Whereas $54.3 \%$ of the control subjects were GSTP1 wild type allele homozygous, 40.0\% were heterozygous and $5.71 \%$ mutant allele homozygous. There was dramatic inverted relation between positive IHC ER staining and increasing grade of tumors in general $(100 \%, 88.6 \%, 40.4 \%)$ and especially among tumors with heterozygote genotype of GSTP1 $(70 \%, 35.4 \%, 22.7)$. There was increase in positive IHC HER2 staining consistent with higher grades in general $(20 \%, 29.6 \%, 50.0 \%)$, especially among tumors with GSTP1 wild allele homozygote genotype $(5.0 \%, 9.1 \%, 31.8 \%)$. A remarkable reverse relation was also observed between the fraction of IHC hormone receptor phenotype ER+/PR+/ HER2- and increased grade of tumors $(60.0 \%$, $\mathbf{4 5 . 5 \%}$, and $27.3 \%$ ) especially among tumors with GSTP1 heterozygote genotype, and a similar link was noted regarding ER+/PR-/ HER2- and tumor grade. There was increase in frequency of ER-/PR-/ HER2- $(0.0 \%, 6.8 \%$, and $18.2 \%)$ and ER-/PR-/ HER2+ $(0.0 \%, 4.54 \%$, and $40.9 \%)$ consistent with the higher grades of tumors in general and especially GSTP1 heterozygote genotype tumors. As a conclusion, there is no correlation between GSTP1 polymorphism and increased risk of breast cancer i.e. the mutant allele is randomly distributed in cancer and control cases. However, there is a link between GSTP1 genotypes and hormone receptor expression status and certain phenotypes of breast cancer, which may have clinical importance.
\end{abstract}

Keywords: Breast cancer - ER - HER2 - PR - GSTP1 - polymorphism - susceptibility

Asian Pac J Cancer Prev, 16 (5), 1707-1713

\section{Introduction}

Cancer of breast is universally ranked first widespread malignancy in female population. In Saudi Arabia, breast cancer has a similar ranking among cancers in female population and accounts for $(25.1 \%)$ of all newly diagnosed female malignant tumors. The rate of crude incidence of female breast carcinoma in Saudi Arabia was (22.7) per 100,000 female population in 2009 . Morphological distribution of female breast cancer showed that invasive or infiltrating ductal carcinoma (IDC) was $(78.2 \%)$, invasive or infiltrating lobular carcinoma (ILC) was (6.3\%), invasive ductal carcinoma mixed with other types $(2.2 \%)$, and $0.9 \%$ represented mixed types of invasive ductal and lobular carcinomas. The remaining were other types of morphology (Al-Eid and Garcia, 2012).

Breast tumors are well known as highly heterogeneous tumors with diverse biological, pathological, clinical characteristics and response to treatment which has been attributed partially to various risk factors including reproductive, genetic, and environmental (Di Cosimo and Baselga, 2010; Ban and Godellas, 2014). Many recent reports have restated its heterogeneity based on molecular and genetic profile and classification (Prat and Perou, 2011; Tamimi et al., 2012). Many classic variables influence breast cancer prognosis and management such as histopathology type of tumor, grade, size, involvement of lymph nodes, immunohistochemistry profile of hormone receptors and, in recent years, status of HER2 (Horita et al., 2001; Kaptain et al., 2001; Sofi et al., 2012; Ng et al., 2014).

Reproductive occasions, which manipulate lifespan quantity of hormones, and oxidative stress, represent the main risk for developing breast cancer (Nourazarian et al., 2014). Free radicals may be produced by several

${ }^{1}$ Department of Pathology, Rabigh Faculty of Medicine, ${ }^{2}$ Department of Medical Laboratory Technology, Faculty of Applied Medical Sciences, ${ }^{3}$ Department of Pathology, Faculty of Medicine, ${ }^{4}$ King Fahd Medical Research Centre, ${ }^{5}$ Department of Family and Community Medicine, Rabigh Faculty of Medicine, King Abdulaziz University, Jeddah, Saudi Arabia *For correspondence: mnkhabaz@kau.edu.sa;nkhabaz@yahoo.co.uk 
mechanisms. Recognition of susceptibility causes, which make persons vulnerable to breast neoplasms when they are subjected to certain environmental elements, might provide additional perception into the causality of this malignancy. Mitrunen and his colleagues proposed that almost eighty percent of tumors occur as a consequence of environmental agents' exposure (Mitrunen et al., 2001). Enzymes engaged in detoxifying carcinogenic agents in addition to DNA reparation may take important part in the breast cancer susceptibility and other types of malignant neoplasms. The detoxifying function efficacy of this type of enzyme is governed by certain genes (Joanne et al., 2000; Hisako et al., 2001; Wu et al., 2002).

Glutathione S-transferases are engaged in the detoxification of reactive metabolites of carcinogens such as polycyclic aromatic hydrocarbons. Furthermore, GSTs are implicated in the metabolism of deferent classes of anticancer drugs (Hamilton et al., 2003). The developmental glutathione S-transferase expression is of interest because these loci demonstrate time- and tissue-specific expression (Helzlsouer et al., 1998; Mohammadzadeha et al., 2003). The coding gene of glutathione S-transferase pi-class (GSTP1) is located on chromosome 11q13. GSTP1 polymorphism may affect the function of enzymes and decreased enzyme activity (Allan et al., 2001). Since a broad panel of organs including placenta, prostate, colon, breast, esophagus, brain, spleen and lung express GSTP1 consistently in their tissues (Welfare et al., 1999), and a wide range of precancerous and neoplastic growths have been shown to overexpress GSTP1, which propose a conceivable GSTP1 polymorphism association in breast cancer etiology (Joanne et al., 2000).

A common functional Ile105Val polymorphism of GSTP1 result from point mutation within nucleotide 313 , which gives rise to an amino acid substitution isoleucine (Ile) to valine (Val) located on the codon 105 of exon 5 (Ile105Val). This change occurs in the binding site of GSTP1 and hence decreases catalytic effort of GSTP1. Change in protein expression may cause ensuing carcinogens buildup in the body, leading to the emergent of a malignant phenotype. The increasing level of environmental pollution and lifestyle changes in countries such as Saudi Arabia may influence breast cancer risk among those carrying GSTP1 105Val allele. DNA alteration in somatic cells due to toxic damage stimulates carcinogenesis and tumors development with aggressive features (Yang et al., 2005). Reduced detoxification of carcinogens or DNA repair as a result of functional polymorphisms remains an attractive assumption (Yang et al., 2001; Das et al., 2004; Eunice et al., 2005; ).

Many studies investigated the possible link between GSTP1 polymorphisms and breast cancer risk, and have shown conflicting results (Gilbert et al., 1993; Silvestrini et al., 1997; Buser et al., 1997; Helzlsouer et al., 1998; Millikan et al., 2000; Mitrunen et al.,2001; Gudmundsdottir et al., 2001; Huang et al., 2003; Egan et al., 2004; Sreenath et al., 2005; Moureau-Zabotto et al., 2006; Unlu et al., 2008; Saxena et al., 2009; Arun et al., 2010; Pongtheerat et al., 2011; Zhang et al., 2011; Ramalhinho et al., 2011; Aguiar et al., 2012; Ramalhinho et al., 2012; Khabaz,
2014; Rodríguez et al., 2014). Many previous studies revealed GSTP1 polymorphism has no correlation with clinical and pathologic tumor characteristics (Buser et al., 1997; Huang et al., 2003) only a few studies showed an inverse correlation with ER (Gilbert et al., 1993; Silvestrini et al., 1997; Moureau-Zabotto et al., 2006). Furthermore, two studies of Pongtheerat and colleagues reported that GSTP1 polymorphism was unrelated to increased risk of breast cancer, but found an inverse relationship between GSTP1 genotype and progesterone receptor protein (Pongtheerat et al., 2009; Pongtheerat et al., 2011), and positive correlation with better tumor differentiation and grade (Cairns et al., 1992; Haas et al., 2006). It is difficult to compare and draw meaningful conclusions from these studies as the patient population and definition of positive GSTP1 results differ from study to study. However, to the best of our knowledge and literature search, none of the published studies investigated the correlation between GSTP1 Ile105Val polymorphism and immunohistochemistry subtypes of breast cancer. Therefore, the present study aims to research the relation between GSTP1 Ile105Val polymorphism and certain hormone receptors phenotypes of breast cancer.

\section{Materials and Methods}

Paraffin embedded tissue samples of eighty six cases of previously diagnosed invasive ductal carcinoma of breast were recruited in this study, in addition to 35 samples of non-cancerous breast tissue as a control group. The patients of this study have undergone breast tumor resections with regional lymph node dissection between January 2010 and June 2012 at the teaching hospital of King Abdulaziz University. Clinical data (age, size and grade of carcinoma) and tissue samples were gathered from the Pathology Department at King Abdulaziz University. All cases which had received radiation therapy or chemotherapy were excluded from this study. All samples were stored at room temperature.

Control group was selected from patients who were biopsied for noncancerous conditions (including fibroadenomas, fibrocystic changes, duct ectasias, sclerosing adenosis, hyperplasias, and intraductal papillomas), as well as nearby normal tissue and distant surgical margins. The mean age is 27.5 years, ranging from 16 to 59 years. The benign lesions were obtained by open surgical biopsy due to the fear of hidden microscopic cancer. All blocks of noncancerous control and tumor tissues were serially sectioned and used in the present study.

The patient's median age was 54.6 years ranging from 28 to 80 years. Lymph node involvement was detected in $48(55.8 \%)$ cases. The average size of tumors was 3.6 $\mathrm{cm}$ (range, 0.4 to $9.5 \mathrm{~cm}$ ). Classification and grading of breast cancer were consistent with WHO categorization of breast tumors (Tavassoli and Devilee, 2003) and modified Nottingham Grading System respectively. The distribution of histoprognostic grades was $23.26 \%$ for type I, $51.16 \%$ for type II, and $25.58 \%$ for type III (Table 1).

\section{Immunohistochemistry (IHC)}


Archive materials of breast cancer cases were obtained initially as paraffin-embedded blocks or surgical specimens, which were formalin fixed, then processed, sectioned and hematoxylin and eosin stained. Hormone receptors and HER2 expression were evaluated using routine IHC. Semi quantitatively measurement were employed for positive ER and PR stained nuclei and HER2 stained membranes. The immunohistochemistry staining patterns were ranked in terms of intensity of stain as follows: +3 strong, +2 moderate, and 0 or +1 no staining or weak. The estimated grade of staining intensity reflected positive stained tumor cells that count more than $10 \%$.

\section{DNA isolation}

Paraffin-embedded tissue samples were used to extract genomic DNA. QIAamp DNA FFPE Kit (Qiagen) was used in accordance to manufacturer's instruction. Purified DNA was eluted in $50 \mu 1$ elution buffer and saved at $-40^{\circ} \mathrm{C}$. Purity and concentration of isolated DNA was analyzed by nanodrop-2000 (Thermo Scientific).

\section{GSTP1 genotyping}

Genotyping for the recognition of GSTP1 polymorphism in Saudi breast cancer patients was performed using commercial kit (Qiagen; Cyber green PCR kit) along with the manufacturer's recommendations. Approximately 200 ng DNA was magnified in an overall volume of $25 \mu 1 /$ reaction. We used GSTP1 oligonucleotide primers (F: 5'-ACCCCAGGGCTCTATGGGAA-3', R: 5'-TGAGGGCACAAGAAGCCCCT-3') from MWG-Biotech to amplify GSTP1 fragment. The PCR amplification reaction was done using a thermocycler 480 (Applied Biosystems) starting with $15 \mathrm{~min}$ at $94^{\circ} \mathrm{C}$ for initial denaturation step, followed by thirty five cycles for denaturation over $1 \mathrm{~min}$ at $94^{\circ} \mathrm{C}$, then annealing at $57^{\circ} \mathrm{C}$ for $1 \mathrm{~min}$, and extension for $1 \mathrm{~min}$ at $74^{\circ} \mathrm{C}$. A final extension for ten min at $72^{\circ} \mathrm{C}$ was followed. The products of PCR were examined using $1 \%$ agarose gel electrophoresis and visualized using UV-transluminator (SYNGENE).

\section{DNA sequencing}

Applied Biosystems Genetic analyzer 3500 and sequencing kit (big dye terminator v3.1) were employed to sequence the amplified PCR products in line with the manufacturer's instruction. The resulting sequence data were studied using Applied Biosystems sequence analysis software (v 5.4). Genotypes were determined as wild type Ile/Ile, heterozygous type Ile/Val or homozygous variant type $\mathrm{Val} / \mathrm{Val}$.

\section{Statistical analyses}

Analyses of the results were completed using SPSS version 20 and Chi-square test to establish any significant differences in polymorphism incidences between breast cancer cases and control group. Calculation of statistics was performed based on $95 \%$ confidence intervals.

Table 1. Clinicopathological Characteristics of Invasive Ductal Carcinoma Patients

\begin{tabular}{|c|c|c|c|c|c|}
\hline \multicolumn{5}{|l|}{ Characteristics } & \multirow{2}{*}{$\frac{\text { Number of patient }}{86}$} \\
\hline Total cases & & & & & \\
\hline \multirow{4}{*}{ Age of patient } & $<40$ & & & & $11(12.79)$ \\
\hline & $40-49$ & & & & $21(24.42)$ \\
\hline & $50-59$ & & & & $29(33.72)$ \\
\hline & $\geq 60$ & & & & $25(29.07)$ \\
\hline Average age & 54.6 & & & & \\
\hline \multirow[t]{2}{*}{ Laterality } & Left & & & & $51(59.3)$ \\
\hline & Right & & & & $35(40.7)$ \\
\hline \multirow[t]{4}{*}{ Size of tumor } & $<1 \mathrm{~cm}$ & & & & 4 (4.6) \\
\hline & $\geq 1$ anc & & & & $32(37.3)$ \\
\hline & $\geq 3$ and & & & & $29(33.7)$ \\
\hline & $\geq 5 \mathrm{~cm}$ & & & & $21(24.4)$ \\
\hline Average size & $3.6 \mathrm{~cm}$ & & & & \\
\hline Lymph node involvement & Yes & & & & $48(55.8)$ \\
\hline \multirow[t]{5}{*}{ Grade } & & AA & AG & GG & \\
\hline & I & $6(15 \%)$ & $14(31.2 \%)$ & 0 & $20(23.26)$ \\
\hline & II & $24(60 \%)$ & $19(42.2 \%)$ & 1 & $44(51.16)$ \\
\hline & III & $10(25 \%)$ & $12(26.6 \%)$ & 0 & $22(25.58)$ \\
\hline & Total & $40(46.51 \%)$ & $45(52.33 \%)$ & $1(1.16 \%)$ & 86 \\
\hline Triple positive & & 5 & 7 & 1 & $13(15.12)$ \\
\hline Triple negative & & 3 & 4 & 0 & $7(8.14)$ \\
\hline ER+/PR+/ HER2- & & 19 & 19 & 0 & $38(44.19)$ \\
\hline ER+/PR-/ HER2+ & & 2 & 2 & 0 & $4(4.65)$ \\
\hline ER+/PR-/ HER2- & & 6 & 7 & 0 & $13(15.12)$ \\
\hline ER-/PR-/ HER2+ & & 5 & 6 & 0 & $11(12.79)$ \\
\hline Total & & 40 & 45 & 1 & 86 \\
\hline $\mathrm{ER}+$ & & $32(37.21 \%)$ & $35(40.7 \%)$ & $1(1.16 \%)$ & 68 \\
\hline ER- & & $8(9.3 \%)$ & $10(11.63 \%)$ & 0 & 18 \\
\hline $\mathrm{PR}+$ & & $24(27.91 \%)$ & $26(30.23 \%)$ & $1(1.16 \%)$ & 51 \\
\hline PR- & & $16(18.61 \%)$ & $19(22.1 \%)$ & 0 & 35 \\
\hline HER2+ & & $12(13.95 \%)$ & $15(17.44 \%)$ & $1(1.16 \%)$ & 28 \\
\hline HER2- & & $28(32.56 \%)$ & $30(34.88 \%)$ & 0 & 58 \\
\hline
\end{tabular}




\section{Results}

The observed percentages of wild allele homozygote, heterozygote and variant allele homozygote genotypes of GSTP1 over invasive ductal carcinomas were $46.51 \%$, $52.33 \%$ and $1.16 \%$ respectively; Whereas, $54.29 \%$ of the control subjects were GSTP1 wild type allele homozygous, $40 \%$ were heterozygous and $5.71 \%$ mutant allele homozygous (Table 2). A small variance was revealed not to be significant. Generally, the outcomes designate that GSTP1 genotypes do not seem to manipulate breast cancer susceptibility in the examined Saudi female population.

Sixty eight (79.07\%), $51(59.3 \%)$, and $28(31.4 \%)$ of invasive ductal carcinomas showed positive IHC stain for ER, PR and HER2 in that order. Eighty six carcinoma cases in the present study showed six hormone receptor IHC phenotypes in descendent order as follows; 38

Table 2. GSTP1 Genotypes of Breast Cancer Cases and Controls

\begin{tabular}{lrrrrl}
\hline GSTP1 genotypes & \multicolumn{2}{c}{ Cancer cases } & \multicolumn{2}{c}{ Controls } & $p$ \\
& Total & \multicolumn{1}{c}{$\%$} & Total & $\%$ & \\
\hline Ile/Ile & 40 & 46.51 & 19 & 54.29 & \\
Ile/Val & 45 & 52.33 & 14 & 40 & 0.307 \\
Val/Val & 1 & 1.16 & 2 & 5.71 & \\
Total & 86 & 100 & 35 & 100 & \\
\hline
\end{tabular}

(44.19\%) ER+/PR+/ HER2-, $13(15.12 \%)$ Triple positive, 13 (15.12\%) ER+/PR-/ HER2-, 11 (12.79\%) ER-/PR-/ HER2+, 7 (8.14\%) Triple negative and 4 (4.65\%) ER+/ PR-/ HER2+ (Table 1). However, in general all differences regarding clinical data, phenotypes and GSTP1 genotypes between tumors are not statistically significant, but may be of clinical importance. The results showed there is dramatic inverted relation between the percentage of positive IHC ER stain and increased grade of tumors in general $(100 \%, 88.64 \%, 40.41)$ and especially among tumors with heterozygote genotype of GSTP1 (70\%, $35.36 \%, 22.72)$, and vice versa $(0 \%, 11.36 \%, 59.09 \%)$ for the percentage of negative IHC ER stain and tumor grade in both wild allele homozygote and heterozygote genotype tumors (Table 3). Similar relation was found in tumors with heterozygote genotype of GSTP1 regarding the percentage of IHC positive and negative PR stain and tumor grade. Whereas, there was increase in the percentage of positive IHC HER2 stain consistent with higher grades in general $(20 \%, 29.55 \%, 50 \%)$ and especially among tumors with wild allele homozygote genotype of GSTP1 $(5 \%, 9.1 \%, 31.82 \%)$, and vice versa $(80 \%, 70.45 \%, 50 \%)$ for the percentage of negative IHC HER2 stain and tumor grade (Table 3).

There was remarkable inverted relation between the percentage of IHC hormone receptor phenotype ER+/ $\mathrm{PR}+/$ HER2- and increased grade of tumor $(60 \%, 45.45 \%$,

Table 3. Relation between Tumor Grades, Immunohistochemistry Phenotypes and GSTP1 Genotypes

\begin{tabular}{|c|c|c|c|c|c|c|c|}
\hline & Total & & $\mathrm{R}+\mathrm{PR}+\mathrm{HER} 2-$ & & ER+PR-HER2+ & & ER-PR-HER2+ \\
\hline & & ER+PR+HER2- & & ER+PR-HER2- & & ER-PR-HER2- & \\
\hline Tumor G & & & & & & & \\
\hline A & $6(30 \%)$ & 3 & 1 & 2 & 0 & 0 & 0 \\
\hline $\mathrm{AG}$ & $14(70 \%)$ & $9(64.28 \%)$ & 2 & 2 & 1 & 0 & 0 \\
\hline $\mathrm{G}$ & 0 & 0 & 0 & 0 & 0 & 0 & 0 \\
\hline Total & 20 & $12(60 \%)$ & $3(15 \%)$ & $4(20 \%)$ & $1(5 \%)$ & 0 & 0 \\
\hline Tumor G & & & & & & & \\
\hline A & $24(54.54 \%)$ & 14 & 2 & 4 & 2 & 2 & 0 \\
\hline $\mathrm{AG}$ & $19(43.18 \%)$ & $6(31.57 \%)$ & 5 & 4 & 1 & $1(5.26 \%)$ & $2(10.52 \%)$ \\
\hline $\mathrm{G}$ & 1 & 0 & 1 & 0 & 0 & 0 & 0 \\
\hline Total & 44 & $20(45.45 \%)$ & $8(18.18 \%)$ & $8(18.18 \%)$ & $3(6.81 \%)$ & $3(6.8 \%)$ & $2(4.54 \%)$ \\
\hline Tumor G & & & & & & & \\
\hline A & $10(45.45 \%)$ & 2 & 2 & 0 & 0 & 1 & 5 \\
\hline $\mathrm{AG}$ & $12(54.55 \%)$ & $4(33.33 \%)$ & 0 & 1 & 0 & $3(25 \%)$ & $4(33.33 \%)$ \\
\hline $\mathrm{G}$ & 0 & 0 & 0 & 0 & 0 & 0 & 0 \\
\hline Total & 22 & $6(27.27 \%)$ & $2(13.64 \%)$ & $1(4.55 \%)$ & 0 & $4(18.18 \%)$ & $9(40.91 \%)$ \\
\hline & & $\mathrm{ER}+$ & ER- & $\mathrm{PR}+$ & PR- & HER2+ & HER2- \\
\hline Tumor G & & & & & & & \\
\hline A & $6(30 \%)$ & $6(30 \%)$ & 0 & $4(20 \%)$ & $2(10 \%)$ & $1(5 \%)$ & $5(25 \%)$ \\
\hline AG & $14(70 \%)$ & $14(70 \%)$ & 0 & $11(55 \%)$ & $3(15 \%)$ & $3(15 \%)$ & $11(55 \%)$ \\
\hline $\mathrm{G}$ & 0 & 0 & 0 & 0 & 0 & 0 & 0 \\
\hline Total & 20 & $20(100 \%)$ & 0 & $15(75 \%)$ & $5(25 \%)$ & $4(20 \%)$ & $16(80 \%)$ \\
\hline Tumor G & & & & & & & \\
\hline A & $24(54.54 \%)$ & $22(50 \%)$ & $2(4.54 \%)$ & $16(36.36 \%)$ & $8(18.18 \%)$ & $4(9.1 \%)$ & $20(45.45 \%)$ \\
\hline $\mathrm{AG}$ & $19(43.18 \%)$ & $16(36.36 \%)$ & $3(6.82 \%)$ & $11(25 \%)$ & $8(18.18 \%)$ & $8(18.18 \%)$ & $11(25 \%)$ \\
\hline G & 1 & $1(2.27 \%)$ & 0 & 1 & 0 & 1 & 0 \\
\hline Total & 44 & $39(88.64 \%)$ & $5(11.36 \%)$ & $28(63.64 \%)$ & $16(36.36 \%)$ & $13(29.55 \%)$ & $31(70.45 \%)$ \\
\hline Tumor G & & & & & & & \\
\hline A & $10(45.45 \%)$ & $4(18.18 \%)$ & $6(27.27 \%)$ & $4(18.18 \%)$ & $6(27.27 \%)$ & $7(31.82 \%)$ & $3(13.64 \%)$ \\
\hline $\mathrm{AG}$ & $12(54.55 \%)$ & $5(22.72 \%)$ & $7(31.82 \%)$ & $4(18.18 \%)$ & $8(36.36)$ & $4(18.18 \%)$ & $8(36.36 \%)$ \\
\hline $\mathrm{G}$ & 0 & 0 & 0 & 0 & 0 & 0 & 0 \\
\hline Total & 22 & $9(40.91 \%)$ & $13(59.09 \%)$ & $8(36.36 \%)$ & $14(63.64 \%)$ & $11(50 \%)$ & $11(50 \%)$ \\
\hline
\end{tabular}


and $27.27 \%$ ) especially among tumor with GSTP1 heterozygote genotype $(64.28 \%, 31.57 \%, 33.33 \%)$, and alike relation was observed regarding ER+/PR-/ HER2and tumor grades (Table 3 ). Whereas, there was increase in frequency of ER-/PR-/ HER2- $(0 \%, 6.8 \%$, and $18.18 \%)$ and ER-/PR-/ HER2+ $(0 \%, 4.54 \%$, and $40.91 \%)$ consistent with the higher grades of tumors in general and especially GSTP1 heterozygote genotype tumors (Table 3).

\section{Discussion}

Immunohistochemical staining of ER, PR and HER2 has been used to classify breast tumors and considered the basis for risk stratification of breast cancer and influences the usage of endocrine therapy (Osborne et al., 1980; Ross et al., 2009; Hammond et al., 2010). Many studies tried to find a potential association between possible susceptibility gene genotypes such as GSTP1 and the clinical, pathological characteristics and hormone receptor expression status in breast cancer. However, to date the results are still somewhat controversial and may vary from population to population. (Gilbert et al., 1993; Silvestrini et al., 1997; Buser et al., 1997; Helzlsouer et al., 1998; Millikan et al., 2000; Mitrunen et al., 2001; Gudmundsdottir et al., 2001; Huang et al., 2003; Egan et al., 2004; Sreenath et al., 2005; Moureau-Zabotto et al., 2006; Unlu et al., 2008; Saxena et al., 2009; Arun et al., 2010; Pongtheerat et al., 2011; Zhang et al., 2011; Ramalhinho et al., 2011; Aguiar et al., 2012; Ramalhinho et al., 2012; Saxena et al., 2013; Khabaz, 2014; Rodríguez et al., 2014).

Several metabolic enzymes which are encoded by polymorphic genes have been suspected to be involved in breast cancer susceptibility (Saxena et al., 2013). Several studies reported that the GSTP1 polymorphism is associated with a higher risk of breast cancer especially Ile/Val and Val/Val allele carriers in different population such as Americans (Kadouri et al., 2008), Indians (Samson et al., 2007; Saxena et al., 2009), Chinese in North china (Ge et al., 2013) and Chinese in Southeast China (Lee et al., 2008). On the other hand, studies of the Finnish (Mitrunen et al., 2001) and Koreans (Kim et al., 2004) revealed that the variant allele of GSTP1 was connected with a reduced breast cancer risk. However, several reports on Jordanian (Khabaz, 2014), African-Americans (Vogl et al., 2004), white women in North Carolina (Millikan et al., 2000), Caucasians (Curran et al., 2000), and the present study on Saudi female population, no association were detected between the polymorphism of GSTP1 and the risk of breast cancer.

Although GSTP1 polymorphism was not related to an augmented breast cancer risk in the present study, it had positive link with better tumor differentiation and grade. In addition, a dramatic inverted relation between positive IHC ER stain and increased grade of tumors in general was present. It was found especially among tumors with heterozygote genotype of GSTP1, and vice versa for negative IHC ER stain and tumor grade in both wild allele homozygote and heterozygote genotype tumors (Table 3). Similar relation was found in tumors with heterozygote genotype of GSTP1 regarding positive and negative IHC PR stain and tumor grade. These results are consistent with studies that showed an inverse correlation with ER (Gilbert et al., 1993; Silvestrini et al., 1997; MoureauZabotto et al., 2006; Rodríguez et al., 2014), and the two studies of Pongtheerat and colleagues which reported GSTP1 polymorphism was unrelated to increased risk of breast cancer, but found an inverse relationship between GSTP1 genotype and progesterone receptor protein (Pongtheerat et al., 2009; Pongtheerat et al., 2011), and positive correlation with better tumor differentiation and grade (Cairns et al., 1992; Haas et al., 2006). The findings of the current study are in harmony with the report of Ge et al who reported that GSTP1 105Val allele carriers were more liable to have a higher histological grade tumor and negative ER stain than Ile/Ile allele carriers (Ge et al., 2013). Our findings also showed an increase in positive IHC HER2 stain consistent with higher grades in general and especially among tumors with wild allele homozygote genotype of GSTP1, and vice versa for negative IHC HER2 stain and tumor grade (Table 3). These findings showed the same trend as earlier results (Pongtheerat et al., 2009; Pongtheerat et al., 2011; Romero et al., 2012).

Furthermore, there was a remarkable inverted relation between the percentage of ER+/PR+/ HER2- phenotype and increased grade of tumor, especially among tumor with GSTP1 heterozygote genotype, and alike relation was observed regarding ER+/PR-/ HER2- and tumor grades (Table 3). Whereas, there was increase in the frequency of ER-/PR-/ HER2- and ER-/PR-/ HER2+ phenotypes consistent with the higher grades of breast tumors in general and especially of those have GSTP1 heterozygote genotype (Table 3). These findings are the first to be reported in the literature to the best of our knowledge and conducted literature search.

However, the present study, which recruited a comparatively small number of controls and breast cancer cases, and other previous studies, make it difficult to compare and draw meaningful conclusions from as the patient population and definition of GSTP1 results differ from study to study. Bearing in mind the heterogeneity of the mutagenic and carcinogenic substances as well as the complex xenobiotic metabolic reaction, larger comprehensive research projects are essential for evaluating the susceptibility to breast cancer. These studies should investigate the interaction between a wide panel of high or low penetrance genes in addition to GSTP1 and environmental exposures.

In conclusion, in general, this study did not confirm the suggestions of previous studies about the positive effects of GSTP1 polymorphisms on breast cancer susceptibility. The isoleucine allele does not seem to have anti-breast cancer development effects. There is no correlation between the presence of the mutant allele and increased breast cancer risk i.e. the mutant allele is randomly distributed in cancer and control cases. However, it does point to a link between GSTP1 genotypes and hormone receptor expression status and certain phenotypes (ER+/ PR+/ HER2-, ER-/PR-/ HER2- and ER-/PR-/ HER2+) of breast cancer. 


\section{Acknowledgements}

This project was funded by the Deanship of Scientific Research, (DSR), King Abdulaziz University (KAU), Jeddah, under grant number (460/828/1432). The authors acknowledge with thanks DSR technical and financial support. The authors would also like to acknowledge with thanks, the support of all administrative and technical staff of King Fahd Center for Medical Research.

\section{References}

Aguiar ES, Giacomazzi J, Schmidt AV, et al (2012). GSTM1, GSTT1, and GSTP1 polymorphisms, breast cancer risk factors and mammographic density in women submitted to breast cancer screening. Rev Bras Epidemiol, 15, 246-255.

Al-Eid HS, Garcia AD (2012). Cancer incidence report Saudi Arabia 2009. Saudi cancer registry, ministry of health, Kingdom of Saudi Arabia.

Allan JM, Wild CP, Rollinson S, et al (2001). Polymorphism in Glutathione S-transferase P1 is associated with susceptibility to chemotherapy-induced leukemia. PNAS, 98, 11592-7.

Arun BK, Granville LA, Yin G, et al (2010). Glutathione-stransferase-pi expression in early breast cancer: association with outcome and response to chemotherapy. Cancer Invest, 28, 554-9.

Ban KA, Godellas CV (2014). Epidemiology of Breast Cancer. Surg Oncol Clin N Am, 3, 409-22.

Buser K, Joncourt F, Altermatt HJ, et al (1997). Breast cancer: pretreatment drug resistance parameters (GSH-system, ATase, P-glycoprotein) in tumor tissue and their correlation with clinical and prognostic characteristics. Ann Oncol, 8, 335-41.

Cairns J, Wright C, Cattan AR, et al (1992). Immunohistochemical demonstration of glutathione S-transferases in primary human breast carcinomas. J Pathol, 166, 19-25.

Curran JE, Weinstein SR, Griffiths LR (2000) Polymorphisms of glutathione S-transferase genes (GSTM1, GSTP1 and GSTT1) and breast cancer susceptibility. Cancer Lett, 153, 113-120.

Das P, Singal R (2004). DNA methylation and cancer. J Clin Oncol, 22, 4632-4642.

Di Cosimo S, Baselga J (2010). Management of breast cancer with targeted agents: importance of heterogeneity. Nat Rev Clin Oncol, 3, 139-47.

Egan KM, Cai Q, Shu XO, et al (2004). Genetic polymorphisms in GSTM1, GSTP1, and GSTT1 and the risk for breast cancer: results from the Shanghai Breast Cancer Study and meta-analysis. Cancer Epidemiol Biomarkers Prev, 13, 197-204.

Eunice C, Shui L, Kin F, Yok K (2005). Polymorphisms of the GSTM1, GSTP1, MPO, XRCC1, and NQO1 genes in Chinese patients with non-small cell lung cancers: relationship with aberrant promoter methylation of the CDKN2A and RARB genes. Cancer Genet Cytogenet, 162, 10-20.

Ge J, Tian AX, Wang QS, et al (2013). The GSTP1 105Val allele increases breast cancer risk and aggressiveness but enhances response to cyclophosphamide chemotherapy in North China. PLoS One, 8, 67589.

Gilbert L, Elwood LJ, Merino M, et al (1993). A pilot study of piclass glutathione $\mathrm{S}$-transferase expression in breast cancer: correlation with estrogen receptor expression and prognosis in node-negative breast cancer. J Clin Oncol, 11, 49-58.

Gudmundsdottir K, Tryggvadottir L, Eyfjord JE (2001). GSTM1, GSTT1, and GST-PI genotypes in relation to breast cancer risk and frequency of mutations in the p53 gene. Cancer Epidemiol Biomarkers Prev, 10, 1169-1173.

Haas S, Pierl C, Harth V, et al (2006). Expression of xenobiotic and steroid hormone metabolizing enzymes in human breast carcinomas. Int J Cancer, 119, 1785-91.

Hamilton DS, Zhang X, Ding Z, et al (2003). Mechanism of the glutathione transferase-catalyzed conversion of antitumor 2-crotonyloxymethyl-2-cycloalkenones to GSH adducts. $J$ Am Chem Soc, 49, 15049-58.

Hammond ME, Hayes DF, Dowsett M, et al (2010). American Society of Clinical Oncology/College of American Pathologists guideline recommendations for immunohistochemical testing of estrogen and progesterone receptors in breast cancer. J Clin Oncol, 28, 2784-95.

Helzlsouer J, Selmin O, Huang Y, et al (1998). Association between glutathione S-transferase M1, P1, and T1 genetic polymorphisms and development of breast cancer. $J$ Natl Cancer Inst, 90, 513-8.

Hisako I, Chikako K, Sachiko S, et al (2001). Glutathione $\mathrm{S}$-transferase polymorphisms and risk of colorectal adenomas. Cancer Lett, 163, 201-6.

Horita K, Yamaguchi A, Hirose K, et al (2001). Prognostic factors affecting disease-free survival rate following surgical resection of primary breast cancer. Eur J Histochem, 1, 73-84.

Huang J, Tan PH, Thiyagarajan J, Bay BH (2003). Prognostic significance of glutathione S-transferase-pi in invasive breast cancer. Mod Pathol, 16, 558-65.

Joanne E. Currana, R. Weinsteinb, Lyn R (2000). Griffithsa, Polymorphisms of glutathione S-transferase genes (GSTM1, GSTP1 and GSTT1) and breast cancer susceptibility, Cancer Lett, 153, 113-120.

Kadouri L, Kote-Jarai Z, Hubert A, et al (2008) GlutathioneS-transferase M1, T1 and P1 polymorphisms, and breast cancer risk, in BRCA1/2 mutation carriers. Br J Cancer, 98, 2006-10.

Kaptain S, Tan LK, Chen B (2001). Her-2/neu and breast cancer. Diagn Mol Pathol, 3, 139-52.

Khabaz MN (2014). Polymorphism of the Glutathione $\mathrm{S}$-Transferase P1 Gene (GST-pi) in breast carcinoma. Pol J Pathol, 65, 141-6.

Kim SU, Lee KM, Park SK, et al (2004). Genetic polymorphism of glutathione S-transferase P1 and breast cancer risk. $J$ Biochem Mol Biol, 37, 582-5.

Lee SA, Fowke JH, Lu W, et al (2008). Cruciferous vegetables, the GSTP1 Ile105Val genetic polymorphism, and breast cancer risk. Am J Clin Nutr, 87, 753-60.

Millikan R, Pittman G, Tse CK, et al (2000). Glutathione S-transferases M1, T1, and P1 and breast cancer. Cancer Epidemiol Biomarkers Prev, 9, 567-73.

Mitrunen K, Jourenkova N, Kataja V, et al (2001). Glutathione S-transferase M1, M3, P1, and T1 genetic polymorphisms and susceptibility to breast cancer. Cancer Epidemiol Biomarkers Prev, 10, 229-36.

Mohammadzadeha S, Nasseri M, Rasaee J, et al (2003). Measurement of glutathione S-transferase and its class- in plasma and tissue biopsies obtained after laparoscopy and endoscopy from subjects with esophagus and gastric cancer. Clin Biochem, 36, 283-88.

Moureau-Zabotto L, Ricci S, Lefranc JP, et al (2006). Prognostic impact of multidrug resistance gene expression on the management of breast cancer in the context of adjuvant therapy based on a series of 171 patients. Br J Cancer, 94 , 473-80.

$\mathrm{Ng} \mathrm{CH}$, Pathy NB, Taib NA, et al (2014). Do clinical features and survival of single hormone receptor positive breast cancers differ from double hormone receptor positive breast cancers? 
Asian Pac J Cancer Prev, 15, 7959-64.

Nourazarian AR, Kangari P, Salmaninejad A (2014). Roles of oxidative stress in the development and progression of breast cancer. Asian Pac J Cancer Prev, 15, 4745-51.

Osborne CK, Yochmowitz MG, Knight WA 3rd, McGuire WL (1980). The value of estrogen and progesterone receptors in the treatment of breast cancer. Cancer, 46, 2884-8.

Pongtheerat T, Pakdeethai S, Purisa W, et al (2011). Promoter methylation and genetic polymorphism of glutathione S-transferase P1 gene (GSTP1) in Thai breast-cancer patients. Asian Pac J Cancer Prev, 12, 2731-4.

Pongtheerat T, Treetrisool M, Purisa W (2009). Glutathione s-transferase polymorphisms in breast cancers of Thai patients. Asian Pac J Cancer Prev, 10, 127-32.

Prat A, Perou CM (2011). Deconstructing the molecular portraits of breast cancer. Mol Oncol, 1, 5-23.

Ramalhinho AC, Fonseca-Moutinho JA, Breitenfeld L (2011). Glutathione S-transferase M1, T1, and P1 genotypes and breast cancer risk: a study in a Portuguese population. $\mathrm{Mol}$ Cell Biochem, 355, 265-271.

Ramalhinho AC, Fonseca-Moutinho JA, Breitenfeld Granadeiro LA (2012). Positive association of polymorphisms in estrogen biosynthesis gene, cyp19a1, and metabolism, GST, in breast cancer susceptibility. DNA Cell Biol, 31, 1100-06.

Rodríguez M, Mejia F, Lecourtois M, Domínguez V, Castillo J (2014). Influence of GSTT1, GSTM1 and GSTP1 polymorphisms on the development of breast cancer. $J$ Cancer Therapy, 5, 552-9

Romero A, Martín M, Oliva B, et al (2012). Glutathione S-transferase $\mathrm{P} 1 \mathrm{c} .313 \mathrm{~A}>\mathrm{G}$ polymorphism could be useful in the prediction of doxorubicin response in breast cancer patients. Ann Oncol, 23, 1750-6.

Ross JS, Slodkowska EA, SymmansWF, et al (2009). The HER-2 receptor and breast cancer: ten years of targeted anti-HER-2 therapy and personalized medicine. Oncologist, 14, 320-68.

Samson M, Swaminathan R, Rama R, et al (2007). Role of GSTM1 (Null/Present), GSTP1 (Ile105Val) and P53 (Arg72Pro) genetic polymorphisms and the risk of breast cancer: a case control study from South India. Asian Pac J Cancer Prev, 8, 253-7.

Saxena A, Dhillon VS, Raish M, et al (2009). Detection and relevance of germline genetic polymorphisms in glutathione S-transferases (GSTs) in breast cancer patients from northern Indian population. Breast Cancer Res Treat, 115, 537-43.

Saxena A, Dhillon VS, Shahid M, et al (2013). GSTP1 methylation and polymorphism increase the risk of breast cancer and the effects of diet and lifestyle in breast cancer patients. Exp Ther Med, 4, 1097-1103.

Silvestrini R, Veneroni S, Benini E, et al (1997). Expression of p53, glutathione S-transferase-pi, and Bcl-2 proteins and benefit from adjuvant radiotherapy in breast cancer. $J$ Nat Cancer Inst, 89, 639-45.

Sofi GN, Sofi JN, Nadeem R, et al (2012). Estrogen receptor and progesterone receptor status in breast cancer in relation to age, histological grade, size of lesion and lymph node involvement. Asian Pac J Cancer Prev, 13, 5047-52.

Sreenath AS, Kumar KR, Reddy GV, et al (2005). Evidence for the association of synaptotagmin with glutathione S-transferases: implications for a novel function in human breast cancer. Clin Biochem, 38, 436-43.

Tamimi RM, Colditz GA, Hazra A, et al (2012). Traditional breast cancer risk factors in relation to molecular subtypes of breast cancer. Breast Cancer Res Treat, 1, 159-67.

Tavassoli FA, Devilee P (2003). Pathology and genetics. in tumors of the breast and female genital organs, WHO Classification of Tumors Series. IARC Press, Lyon, France, pp. 32-34.
Unlu A, Ates NA, Tamer L, et al (2008). Relation of glutathione S-transferase T1, M1 and P1 genotypes and breast cancer risk. Cell Biochem Funct, 26, 643-647.

Vogl FD, Taioli E, Maugard C, et al (2004). Glutathione S-transferases M1, T1, and P1 and breast cancer: a pooled analysis. Cancer Epidemiol Biomarkers Prev, 13, 1473-9.

Welfare M, Adeokun M, Margaret F, Bassendine, Ann D (1999). Polymorphisms in GSTP1, GSTM1, and GSTT1 and susceptibility to colorectal cancer. Cancer Epidemiol Biomarkers Prev, 8, 289-92.

Wu MS, Chen CJ, Lin MT, et al (2002). Genetic polymorphisms of cytochrome P4502E1, glutathione S-transferase M1 and $\mathrm{T} 1$, and susceptibility to gastric carcinoma in Taiwan. Int $J$ Colorectal Dis, 17, 338-43.

Yang G, Shu XO, Ruan ZX, et al (2005). Genetic polymorphisms in glutathione-S-transferase genes (GSTM1, GSTT1, GSTP1) and survival after chemotherapy for invasive breast carcinoma. Cancer, 103, 52-8.

Yang X, Yan L, Davidson E (2001). DNA methylation in breast cancer. Endocr Relat Cancer, 8, 115-127.

Zhang B, Beeghly-Fadiel A, Lu W, et al (2011). Evaluation of functional genetic variants for breast cancer risk: results from the Shanghai breast cancer study. Am J Epidemiol, 173, $1159-70$ 